\title{
Hot Nuclear Matter Equation of State with a Three-body Force
}

\author{
W. Zuo ${ }^{1,2,3}$, Z.H. Li ${ }^{1,2}$, A. $\mathrm{Li}^{3}$, G.C. $\mathrm{Lu}^{1,2}$ \\ ${ }^{1}$ Institute of Modern Physics, Chinese Academy of Sciences, Lanzhou 730000, P.R. China \\ ${ }^{2}$ Graduate School of Chinese Academy of Sciences, Beijing 100039, P. R. China \\ ${ }^{3}$ School of Physics and Technology, Lanzhou University, Lanzhou 730000, P. R. China
}

\begin{abstract}
The finite temperature Brueckner-Hartree-Fock approach is extended by introducing a microscopic three-body force. In the framework of the extended model, the equation of state of hot asymmetric nuclear matter and its isospin dependence have been investigated. The critical temperature of liquid-gas phase transition for symmetric nuclear matter has been calculated and compared with other predictions. It turns out that the three-body force gives a repulsive contribution to the equation of state which is stronger at higher density and as a consequence reduces the critical temperature of liquid-gas phase transition. The calculated energy per nucleon of hot asymmetric nuclear matter is shown to satisfy a simple quadratic dependence on asymmetric parameter $\beta$ as in the zero-temperature case. The symmetry energy and its density dependence have been obtained and discussed. Our results show that the three-body force affects strongly the high-density behavior of the symmetry energy and makes the symmetry energy more sensitive to the variation of temperature. The temperature dependence and the isospin dependence of other physical quantities, such as the proton and neutron single particle potentials and effective masses are also studied. Due to the additional repulsion produced by the three-body force contribution, the proton and neutron single particle potentials are correspondingly enhanced as similar to the zerotemperature case.
\end{abstract}

PACS numbers: 21.65.+f, 13.75.Cs, 24.10.Cn, 05.70.Ce

Typeset using REVTEX 


\section{INTRODUCTION}

One of the most important motivations of heavy ion physics is to investigate the properties of nuclear matter under extreme conditions of temperature, density and isospin. The equation of state (EOS) of asymmetric nuclear matter at finite temperature plays an important role in understanding the dynamics of heavy ion collisions at intermediate and high energies. Theoretical simulations [1] indicate that the main reaction dynamics and observables including collective flow, balance energy, isospin equilibrium, pre-equilibrium nucleon emission, isotopic scaling and isospin diffusion are quite sensitive to the isospin dependent part of the nuclear EOS, especially to the density dependence of the symmetry energy. The properties of hot and dense asymmetric nuclear matter are also of great interest in astrophysics especially in connection with the dynamics of supernova explosions and the thermal evolution of neutron stars [2]. The temperature and isospin dependence of the EOS of asymmetric nuclear matter is crucial for a reliable description of the thermodynamical evolution and the structure of the newborn neutron star ( protoneutron star ) formed in the latest stage of a type-II supernova collapse [2]. Motivated by the general significance in heavy ion physics and astrophysics, the thermodynamical properties of nuclear matter has been investigated extensively within various theoretical models such as the non-relativistic Hartree-Fock approach [3] and the relativistic mean field theory (RMT) [4,5].

Due to the Van der Waals nature of the nucleon-nucleon (NN) interaction, it is generally expected that nuclear matter is likely to exhibit a liquid-gas phase transition [6]. Theoretically, considerable effort has been devoted to establishing the EOS of hot symmetric nuclear matter and discussing the critical phenomena [7-13]. Although almost all the predicted EOSs of infinite symmetric nuclear matter display a typical Van der Waals behavior, the obtained values of the critical temperature $T_{C}$ for the phase transition are distributed in a wide range from $8 \mathrm{MeV}$ to $20 \mathrm{MeV}$. Within the non-relativistic framework, the calculated value of $T_{C}$ for infinite symmetric nuclear matter is $15-20 \mathrm{MeV}[9,10]$ depending on the choice of the theoretical models and the NN interactions. The RMT theory gives a critical temperature of about $14 \mathrm{MeV}[5]$. The value of $T_{C}$ predicted from the Dirac-Brueckner ( DB ) approach is as low as $\sim 8 \mathrm{MeV}$ in Ref. [12] and about $10 \mathrm{MeV}$ in Ref. [13].

On a microscopic basis, the properties of hot asymmetric nuclear matter have been studied in Ref. [14] in the framework of the finite temperature Brueckner-Hartree-Fock ( FTBHF ) approach using the separable version of the Paris two-body force. As well 
known, three-body forces are necessary for reproducing the empirical saturation properties

of cold symmetric nuclear matter in a non-relativistic microscopic approach [15-19]. Two kinds of three-body force ( TBF ) have been adopted in the Brueckner-Hartree-Fock ( BHF ) formalism [15-18]. One is the semi-phenomenological TBF [20] which has two or few adjustable parameters determined by fitting the empirical saturation density and energy of the cold symmetric nuclear matter in the BHF calculations [18]. The other is the microscopic TBF based on meson exchange coupled to the intermediate virtual excitations of nucleonantinucleon pairs and nucleon resonances [15-17].

In the present paper, our aim is to extend the FTBHF approach by introducing the microscopic TBF and to investigate the properties of hot asymmetric nuclear matter. Special attention has been payed on the effects of the TBF contribution and the temperature dependence. The two-body realistic NN interaction adopted in the present calculations is the Argonne $V_{18}\left(A V_{18}\right)$ potential [21]. Our calculations indicate that the TBF affects not only the EOS of hot asymmetric nuclear matter and the critical temperature of the liquid-gas phase transition, but also the single-particle ( s.p. ) properties such as the proton and neutron s.p. potentials and effective masses. The present paper is organized as follows. We will describe briefly the model in Section II. The numerical results are presented and discussed in Section III. In Section IV a summary of the present work is given.

\section{THEORETICAL MODEL}

In general, three parameters are required to specify a given thermodynamical state of hot asymmetric nuclear matter, i.e., the total nucleon number density $\rho$, the isospin asymmetry parameter $\beta=\frac{\rho_{n}-\rho_{p}}{\rho}$ and the temperature $T$. At zero-temperature, the proton and the neutron Fermi momenta are given by $k_{F}^{\tau}=\left[3 \pi^{2} \rho_{\tau}\right]^{1 / 3}$ with $\tau=p$ or $\tau=n$, where $\rho_{n}=\frac{1}{2}$ $(1+\beta) \rho$ and $\rho_{p}=\frac{1}{2}(1-\beta) \rho$ are the proton and neutron number densities, respectively. The formalism of the Brueckner-Bethe-Goldstone (BBG) theory for cold asymmetric nuclear matter can be found in Ref. [22]. The extension to finite temperature is given in Ref. [14]. In the following, we give a brief review for completeness. The starting point of the BBG approach is the Brueckner reaction matrix $G$ which satisfies the following generalized Bethe-Goldstone equation,

$$
G_{\tau, \tau^{\prime}}(\rho, \beta, T, \omega)=v+v \sum_{k_{1} k_{2}} \frac{\left|k_{1} k_{2}\right\rangle Q_{\tau, \tau^{\prime}}\left\langle k_{1} k_{2}\right|}{\omega-e_{\tau}\left(k_{1}\right)-e_{\tau^{\prime}}\left(k_{2}\right)} G_{\tau, \tau^{\prime}}(\rho, \beta, T, \omega)
$$


where $v=v_{2}+V_{3}^{\text {eff }}$ is the nucleon-nucleon (NN) interaction and $\omega$ is the starting energy. In the present calculations the Argonne $V_{18}\left(A V_{18}\right)$ potential [21] is adopted as the bare two-body force $v_{2}$ and $V_{3}^{\text {eff }}$ is the TBF contribution given by Eq.(7)(see below). The finite temperature Pauli operator $Q_{\tau, \tau^{\prime}}$ is simply an extension of the zero-temperature one, i.e,

$$
Q_{\tau, \tau^{\prime}}=Q_{\tau, \tau^{\prime}}\left(k_{1}, k_{2}, \rho, \beta, T\right)=\left[1-f_{\tau}\left(k_{1}, \rho, \beta, T\right)\right]\left[1-f_{\tau^{\prime}}\left(k_{2}, \rho, \beta, T\right)\right]
$$

At finite temperature the Fermi distribution is expressed as,

$$
f_{\tau}(k, \rho, \beta, T)=\left[1+\exp \left(\frac{e_{\tau}(k)-\mu_{\tau}}{T}\right)\right]^{-1}
$$

where $\mu_{\tau}=\mu_{\tau}(k, \rho, T)$ refers to the chemical potential and $e_{\tau}(k)$ is the s.p. energy. For any given density and temperature, we can calculate the chemical potential $\mu_{\tau}$ from the following implicit equation self-consistently by iteration,

$$
\rho_{\tau}=\frac{1}{V} \sum_{k} f_{\tau}(k, \rho, \beta, T)=\frac{1}{V} \sum_{k}\left[1+\exp \left(\frac{e_{\tau}(k)-\mu_{\tau}}{T}\right)\right]^{-1}
$$

The s.p. energy is given by,

$$
e_{\tau}(k) \equiv e_{\tau}(k, \rho, \beta, T)=\frac{\hbar^{2} k^{2}}{2 m}+U_{\tau}(k, \rho, \beta, T)
$$

where $U_{\tau}(k, \rho, \beta, T)$ is the proton or neutron s.p. potential. Within the FTBHF framework the s.p. potential is calculated from the on-shell anti-symmetrized $G$ matrix,

$$
U_{\tau}(k, \rho, \beta, T)=\frac{1}{2} \sum_{\tau^{\prime}} \sum_{\vec{k}^{\prime}} f_{\tau^{\prime}}\left(k^{\prime}, \rho, \beta, T\right)\left\langle k k^{\prime}\left|G_{\tau, \tau^{\prime}}\left(\rho, \beta, T, e_{\tau}(k)+e_{\tau^{\prime}}\left(k^{\prime}\right)\right)\right| k k^{\prime}\right\rangle_{A}
$$

In the present calculations, we adopt the continuous choice [23] for the s.p. potential. On the one hand, the continuous choice has been shown to provide a much faster convergence of the hole-line expansion for the energy per nucleon in nuclear matter at $T=0$ than the gap choice [24]. On the other hand, in the continuous choice, the s.p. potential describes physically the nuclear mean field felt by a nucleon in nuclear medium.

The microscopic TBF adopted in the present calculations is constructed from the mesonexchange current approach [15]. Its components are depicted diagrammatically in Fig. 1, taken from Ref. [15]. Four important mesons $\pi, \rho, \sigma$ and $\omega$ are considered [19]. The TBF contains the contribution of the two-meson exchange part of the NN interaction mediummodified by the intermediate virtual excitation of nucleon resonances, the term associated 
to the non-linear meson-nucleon coupling required by the chiral symmetry, the simplest contribution rising from meson-meson interaction and finally, the two-meson exchange diagram with the virtual excitations of nucleon-antinucleon pairs. The meson masses in the TBF have been fixed at their physical values except for the virtual $\sigma$-meson mass which has been fixed at $540 \mathrm{MeV}$ according to Ref. [15]. This value has been checked to satisfactorily reproduce the $A V_{18}$ interaction from the one-boson-exchange potential (OBEP) model [17]. The other parameters of the TBF, i.e., the coupling constants and the regular masses (form factors), have been determined from the OBEP model to meet the self-consistent requirement with the adopted $A V_{18}$ two-body force. For a more detailed description of the model and of the approximations we refer to Refs. [15-17].

The TBF effect is included in the self-consistent Brueckner procedure along a similar way to the zero temperature case $[15,16]$, where an effective two-body interaction is constructed to avoid the full three-body problem. A detailed description and justification of the procedure can be found in Ref. [15]. Being Extended to finite temperature, the effective two-body interaction $V_{3}^{\text {eff }}(T)$ in $r$-space is given by,

$$
\begin{aligned}
\left\langle\vec{r}_{1}^{\prime} \vec{r}_{2}^{\prime}\left|V_{3}^{\text {eff }}(T)\right| \vec{r}_{1} \vec{r}_{2}\right\rangle & =\frac{1}{4} \operatorname{Tr} \sum_{k_{n}} f\left(k_{n}, \rho, \beta, T\right) \int \mathrm{d} \vec{r}_{3} \mathrm{~d} \vec{r}_{3}^{\prime} \phi_{n}^{*}\left(\vec{r}_{3}^{\prime}\right)\left(1-\eta\left(r_{13}^{\prime}, T\right)\right)\left(1-\eta\left(r_{23}^{\prime}, T\right)\right) \\
& \times W_{3}\left(\vec{r}_{1}^{\prime} \vec{r}_{2}^{\prime} \vec{r}_{3}^{\prime} \mid \vec{r}_{1} \vec{r}_{2} \vec{r}_{3}\right) \phi_{n}\left(r_{3}\right)\left(1-\eta\left(r_{13}, T\right)\right)\left(1-\eta\left(r_{23}, T\right)\right)
\end{aligned}
$$

where the trace is taken with respect to the spin and isospin of the third nucleon. The function $\eta(r, T)$ is the correlation function ( or called defect function ) [25]. The defect function is directly related to the solution of the finite-temperature Bethe-Goldstone equation, i.e. eq.(1) $[23,25]$ and depends on temperature. The transformation of the TBF to the above effective interaction entails a self-consistent coupling between the TBF and the Brueckner procedure of solving the Bethe-Goldstone equation. One first calculates the correlation function with only the two-body force and then builds up the effective interaction $V_{3}^{\text {eff }}(T)$ which in turn is added to the bare two-body force, and again evaluates the correlation function and so on up to convergence is reached.

It is worth to noticing that the TBF itself is the same as that adopted in the zero-temperature case [17] and independent of temperature. As compared to the zerotemperature case, the effective two-body force $V_{3}^{\text {eff }}(T)$ constructed from the TBF depends on temperature due to the medium effects and consequently its effects are expected to be more pronounced at finite temperature. It is obviously from Eq.(7) that the temperature 
dependence of $V_{3}^{\text {eff }}(T)$ comes from the Fermi distribution $f(k, \rho, \beta, T)$ and the defect function $\eta(r, T)$. This temperature dependence is somewhat similar to that of the Skyrme force where a three-body part is equivalent to a two-body density-dependent effective interaction which depends implicitly on temperature via density [11].

We solve Eqs.(1), (4), (5), (6), (7) self-consistently to get the $G$-matrix for any given density, temperature and isospin asymmetry. In general, five iterations is needed to reach a satisfactory convergence. The total energy $E(\rho, \beta, T)=E_{k i n}(\rho, \beta, T)+E_{\text {pot }}(\rho, \beta, T)$ can be obtained from the $G$-matrix. The total kinetic energy is

$$
E_{k i n}(\rho, \beta, T)=\sum_{\tau} \sum_{k} f_{\tau}(k, \rho, \beta, T) \frac{\hbar^{2} k^{2}}{2 m}
$$

and the total potential energy

$$
E_{p o t}(\rho, \beta, T)=\frac{1}{2} \sum_{\tau, \tau^{\prime}} \sum_{k, k^{\prime}} f_{\tau}(k, \rho, \beta, T) f_{\tau^{\prime}}\left(k^{\prime}, \rho, \beta, T\right)\left\langle k k^{\prime}\left|G_{\tau, \tau^{\prime}}\left(\rho, \beta, T, e(k)+e\left(k^{\prime}\right)\right)\right| k k^{\prime}\right\rangle_{A}
$$

The total entropy $S$ can be evaluated in the approximation of a non-interacting Fermi gas of quasi-particle in the mean field $U_{\tau}(k, \rho, \beta, T)[14]$. The free energy and the pressure can be obtained according to the standard thermodynamic relation, i.e,

$$
\begin{gathered}
F=E-T S \\
P=\rho^{2}\left(\frac{\partial F}{\partial \rho}\right)_{T, \beta}
\end{gathered}
$$

Based on the above extended FTBHF approach including the TBF, we can investigate the thermodynamic properties of hot asymmetric nuclear matter at various values of density, temperature and asymmetry.

\section{NUMERICAL RESULTS AND DISCUSSIONS}

The EOS of symmetric nuclear matter at finite temperature is reported in Fig.2, where the isotherms of pressure versus density is given for six values of temperature $T=0,8,10,12,14,16 \mathrm{MeV}$ from the bottom to the top. In the figure the solid and dashed curves indicate the results obtained by using the $A V_{18}$ two-body force plus the TBF and the pure $A V_{18}$ two-body force, respectively. As expected, the TBF gives a repulsive contribution to the EOS of nuclear matter. This contribution becomes stronger as increasing 
density and makes the EOS at high density much stiffer. At zero temperature, the additional repulsion from the TBF leads to a decisive improvement of the calculated saturation density of symmetric nuclear matter towards the empirical value ( comparing the lowest solid and dashed curves ). It is clearly seen from the figure that in both cases with and without the TBF, the predicted EOS exhibits a typical Van der Waals structure in agreement with the results from the Skyrme-Hartree-Fock calculations [9] and from the FTBHF approach based on two-body NN interactions [26], which indicates that the infinite nuclear matter can undergo a liquid-gas phase transition. Without the TBF, the critical temperature $T_{C}$ of the liquid-gas transition is approximately $16 \mathrm{MeV}$. Inclusion of the TBF reduces the critical temperature to about $13 \mathrm{MeV}$ which is smaller than the values $T_{C}=15-20 \mathrm{MeV}$ from the Skryme-Hartree-Fock calculations [9]. The TBF reduction of the critical temperature is readily understood since the TBF effect becomes more pronounced as increasing density and temperature. In finite nuclei, the inclusion of the finite size effects and the Coulomb force may lead to a considerable reduction of $T_{C}$ as discussed in Ref. [11]. The present value $T_{C} \simeq 13 \mathrm{MeV}$ is comparable with the value $T_{C} \simeq 14 \mathrm{MeV}$ predicted by the relativistic mean field theory [5] but it is larger than the ones obtained from the Dirac-Brueckner ( DB ) approach, $T_{C} \simeq 8 \sim 9 \mathrm{MeV}$ in Ref. [12] and $T_{C} \simeq 10 \mathrm{MeV}$ in Ref. [13]. One possible reason for this discrepancy is the difference between the relativistic effect in the DB approach [27] and the effect of the present microscopic TBF as discussed in Ref. [17].

In Fig.3 is reported the proton or neutron s.p. potential in symmetric nuclear matter at a fixed density $\rho=0.16 \mathrm{fm}^{-3}$ for three different values of temperature $T=0,10$ and $20 \mathrm{MeV}$. As increasing temperature, the s.p. potential becomes more repulsive, resulting in an enhancement of the potential part of the energy per nucleon in nuclear matter at finite temperature. It is also seen that the curvature around the Fermi momentum becomes more smooth as increasing temperature. This is in agreement with the prediction obtained by using only pure two-body forces [26] and is attributed to the thermal excitations around the Fermi surface at finite temperature.

Due to the isospin effect, the proton and neutron s.p. potentials are different in asymmetric nuclear matter. In order to discuss the TBF effects on the isospin dependence of the proton and neutron s.p. potentials, in Fig.4 is depicted the proton and neutron s.p. potentials at momentum $k=0$ as a function of asymmetry parameter for $\rho=0.16 \mathrm{fm}^{-3}$ and $T=10 \mathrm{MeV}$. In the figure, the solid and dashed curves indicate the results obtained by 
adopting the $A V_{18}$ plus the TBF and the pure $A V_{18}$ two-body force, respectively. It is clear from the figure that the inclusion of the TBF in the calculations leads to an additional repulsion for both the proton and neutron s.p. potentials in the whole isospin asymmetry range $0 \leq \beta \leq 1$. As similar to the zero-temperature case, the proton s.p. potential becomes more attractive while the neutron one becomes more repulsive going from symmetric $(\beta=0)$ to neutron $(\beta=1)$ matter. Such a different isospin dependence of the proton and neutron s.p. potentials stems mainly from the attractive contribution of the isospin singlet $S D$ tensor channel which becomes stronger for protons and weaker for neutrons as increasing neutron excess [22]. As compared to the two-body force predictions, the TBF contribution shift the proton and neutron s.p. potentials to higher values. In both cases with and without the TBF contribution, the dependence of the proton and neutron s.p. potentials on the asymmetry parameter $\beta$ is almost linear as the same as in the zero-temperature case, which supports microscopically the Lane assumption [28] and extends its validity to the case of finite temperature.

In heavy-ion collisions using neutron-rich nuclei, hot nuclear matter of high isospin asymmetry could be formed and the reaction dynamics is expected to be very sensitive to the density and temperature dependence of the symmetry energy. In the prompt explosion model of a type-II supernova the $\beta$-capture process drives the star to the last stage of the collapse where the proton fraction is about 0.33 and the temperature of a few tens of $\mathrm{MeV}$ may be reached. Therefore, the understanding of the cooling mechanism of a protoneutron star requires the information of the symmetry energy in hot nuclear matter with high accuracy $[29,30]$.

In Fig. 5 is depicted the shift of the energy per nucleon between asymmetric nuclear matter and symmetric nuclear matter, $E_{A}(\rho, \beta, T)-E_{A}(\rho, \beta=0, T)$, versus $\beta^{2}$ at $T=$ $20 \mathrm{MeV}$ for two different densities $\rho=0.16$ and $0.32 \mathrm{fm}^{-3}$. It is shown that the energy per nucleon of asymmetric nuclear matter at finite temperature fulfills a quadratic dependence on asymmetry parameter $\beta$ in the whole asymmetry range $(0 \leq \beta \leq 1)$ as the same as in the case for zero temperature $[22,31,32]$. The above $\beta^{2}$-law comes originally from the empirical mass formula for small isospin asymmetry. The present calculations show that the validity of the empirical $\beta^{2}$-law can be extended to the highest isospin asymmetry and to the case of finite temperature.

Besides the $\beta^{2}$-dependent term, an additional binding term called Wigner energy has 
been found for the $N=Z$ nuclei [33]. The Wigner energy is linear in $\beta$ and manifests itself as a spike in the experimental isobaric mass parabola. The Wigner energy has been explored and discussed extensively in the literatures [34]. It is generally expected that the Wigner energy originates from the neutron-proton $(n p)$ pairing correlations and is the strongest prefigure of the $n p$ paring in the $N=Z$ nuclei [35]. In the present calculations, we do not consider the influence of the $n p$ pairing correlations. Since the $n p$ pairing goes to vanishing very rapidly as soon as the isospin asymmetry and the temperature deviates from zero [36], we expect that the effect of the $n p$ pairing correlation shall be appreciable only for the cold symmetric nuclear matter, which is found to slightly lower the energy per nucleon of the cold symmetric nuclear matter by a self-consistent treatment of the BCS gap equation and the BHF equations [37].

The symmetry energy is defined as

$$
E_{\mathrm{sym}}(\rho, T)=\frac{1}{2}\left[\frac{\partial^{2} E_{A}(\rho, \beta, T)}{\partial \beta^{2}}\right]_{\beta=0}
$$

Due to the simple quadratic dependence of the energy per nucleon on asymmetry parameter, the symmetry energy can be equivalently calculated as the difference between the energy per nucleon of pure neutron matter and symmetric nuclear matter, i.e.,

$$
E_{\text {sym }}(\rho, T)=E_{A}(\rho, \beta=1, T)-E_{A}(\rho, \beta=0, T)
$$

To see the influence of temperature on the symmetry energy, in the left panel of Fig.6 we show the density dependence of the symmetry energy for three different temperatures $T=$ 0,10 and $20 \mathrm{MeV}$. It is seen that the symmetry energy decreases as increasing temperature and at finite temperature it remains a monotonic increasing function of density. As compared to the result by using only a two-body force [38], the inclusion of the TBF contribution enlarges the sensitivity of the symmetry energy with respect to the variation of temperature, especially at high densities. For a given temperature, the TBF contribution leads to a strong enhancement of the symmetry energy at high densities as shown in the right panel of Fig. 6 where the results for $T=20 \mathrm{MeV}$ obtained by using the $A V_{18}$ plus the TBF ( solid curve ) and without the TBF ( dashed curve ) are plotted. It is also seen that the symmetry energy predicted by including the TBF contribution rises much more steeply with density than the corresponding two-body force prediction.

Before summary, we discuss briefly the temperature dependence of the effective mass and 
the TBF effect on the effective mass. The effective mass $m_{\tau}^{*}$ for neutron $(\tau=\mathrm{n})$ or proton $\tau=\mathrm{p}$ is defined as $[14]$

$$
\frac{m_{\tau}^{*}(k)}{m}=\frac{k}{m}\left(\frac{d e_{\tau}(k)}{d k}\right)^{-1}
$$

where $e_{\tau}(k)$ is the neutron or proton s.p. energy. The effective mass describes the nonlocal part of the nuclear mean field which makes the local part less attractive for a nucleon travelling with momentum $k>0$. In Fig.7 is shown the momentum dependence of $m^{*}$ for symmetric nuclear matter at a fixed density $\rho=0.16 \mathrm{fm}^{-3}$ and three different values of temperature $T=0,10$ and $20 \mathrm{MeV}$. The results are comparable with the ones reported in Refs. $[39,40]$. As similar to the zero-temperature case, the momentum dependence of $m^{*}(k)$ is characterized by a wide bump around the Fermi momentum due to the high probability for particle-hole excitations nearby Fermi surface [23]. However as the temperature increases, the peak of $m^{*}$ becomes flatter and the peak value of $m^{*}$ becomes lower. These results

are in agreement with the previous calculations by adopting only two-body forces $[14,25,26]$ and related directly with the temperature effect on the s.p. potential around the Fermi momentum.

To see the isospin effects, in Fig. 8 we display the neutron and proton effective masses at their respective Fermi momenta $k_{F}^{\mathrm{n}}$ and $k_{F}^{\mathrm{p}}$ for $T=10 \mathrm{MeV}$ as a function of asymmetry parameter $\beta$. In the figure, the solid curves are calculated by using the $A V_{18}$ plus the TBF, while the dashed curves by adopting only the $A V_{18}$ two-body force. In both cases with and without including the TBF contribution, the neutron effective mass $m_{n}^{*}$ increases and the proton one $m_{p}^{*}$ decreases as increasing $\beta$. The TBF effect is to shift $m_{n}^{*}$ and $m_{p}^{*}$ to slightly higher values. One can also see from the figure that the linear scissor-shaped behavior predicted for $T=0[22]$ remains unchanged in the case for finite temperature.

\section{SUMMARY AND CONCLUSION}

In summary, we have introduced the TBF contribution into the FTBHF approach and investigated the EOS of asymmetric nuclear matter at finite temperature. By using the extended model, we find that the TBF contribution to the EOS is repulsive. The TBF repulsion increases rapidly as increasing density and consequently the high-density EOS including the TBF contribution becomes much stiffer as compared to the one obtained by adopting only the two-body force. Within the extended model, the calculated EOS of hot 
symmetric nuclear matter exhibits a typical Van der Waals structure, implying the presence of a liquid-gas phase transition. The obtained critical temperature of the liquid-gas phase transition is roughly $16 \mathrm{MaV}$ in the case without including the TBF contribution. The TBF leads to a $3 \mathrm{MeV}$ reduction of the critical temperature to about $13 \mathrm{MeV}$ which is between $T_{C}=15-20 \mathrm{MeV}$ from the non-relativistic approaches and $T_{C} \simeq 10 \mathrm{Mev}$ from the DiracBrueckner method.

Our calculations show that the simple $\beta^{2}$-law fulfilled by the energy per nucleon of asymmetric nuclear matter at $T=0$ can be extended to the case of finite temperature. This is of interest, since it means that the energy per nucleon of hot asymmetric nuclear matter can be extracted from the two limiting cases of symmetric nuclear matter and pure neutron matter. The symmetry energy has been calculated. For a given temperature, the symmetry energy is a monotonic increasing function of density in both cases with and without the TBF. At a fixed density, the symmetry energy decreases as the temperature increases. The TBF gives a strong enhancement of the symmetry energy at high density and makes the symmetry energy rise much more rapidly as compared to the results without including the TBF contribution.

The temperature dependence and the isospin dependence of the proton and neutron s.p. potentials and effective masses have also been calculated and discussed. As expected, the s.p. potential becomes more repulsive and the peak of the effective mass around the Fermi momentum becomes flatter as increasing temperature. It is shown that the neutron effective mass increases and the proton one decreases as increasing $\beta$. Both the TBF contribution and the finite temperature effect do not change this isospin behavior of the neutron and proton effective masses.

Our results show that the TBF contribution affects considerably the stiffness of the EOS of hot nuclear matter and the symmetry energy at high density, and as a consequence, it may have important implication for the investigations of the dynamics evolution of supernova explosions and the structure of protoneutron stars.

\section{ACKNOWLEDGMENT}

The work is supported in part by the Knowledge Innovation Project of the Chinese Academy of Sciences ( KJCX2-SW-N02 ), the Major State Basic Research Development Program of China ( G2000077400 ), and the Major Prophase Research Project of Funda- 
mental Research of the Ministry of Science and Technology of China ( 2002CCB00200 ), the National Natural Science Foundation of China (10235030, 10175082 ). 


\section{REFERENCES}

[1] B. A. Li, C. M. Ko, and W. Bauer, Int. J. Mod. Phys. E7, 147 (1998); M. B. Tsang, W. A. Friedman, C. K. Gelbke, W. G. Lynch, G. Verde, and H. Xu, Phys. Rev. Lett. 86, 5023 (2001).

[2] M. Prakash, I. Bombaci, M. Prakash, P. J. Ellis, J. M. Lattimer, and R. Knorren, Phys. Rep. 280, 1 (1997); H. A. Bethe, Rev. Mod. Phys. 62, 801 (1990); J. A. Pons, S. Reddy,M. Prakash, J. M. Lattimer, and J. A. Miralles, Atrophys. J. 513, 780 (1999); K. Strobel, C. Sohaab, M. K. Weigel, Astron. Astrophys. 350, 497 (1999); G. F. Marranghello, C. A. Z. Vasconcellos, M. Dilling, Int. J. Mod. Phys. E11, 83 (2002).

[3] J. M. Lattimer and D. G. Ravenhall, Astrophys. J, 223, 314 (1978); D. Q. Lamb, J. M. Lattimer, C. J. Pethick, and D. G. Ravenhall, Nucl. Phys. A360, 459, (1981); J. M. Lattimer, C. J. Pethick, D. G. Ravenhall, and D. Q. Lamb, Nucl. Phys. A432, 646, (1985); B. A. Li and C. M. Ko, Nucl. Phys. A618, 498 (1997); D. Catalano, G. Giansiracusa, and U. Lombardo, Nucl. Phys. A681, 390c (2001).

[4] J. D. Walecka, Ann. Phys. 83, 491 (1974); R. A. Freedman, Phys. Lett. B71, 369 (1977).

[5] N. K. Glendenning, Nucl. Phys. A469, 600 (1987); H. Müller and B. D. Serot, Phys. Rev. C52, 2072(1995).

[6] G. Bertsch and P. J. Siemens, Phys. Lett. B126, 9 (1983).

[7] W. A. Küpper, G. Wegmann, and E. R. Hilf, Ann. Phys. 88, 454 (1974).

[8] L. Satpathy, M. Mishra, and R. Nayak, Phys. Rev. C39, 162 (1989).

[9] H. R. Jaqaman, A. Z. Mekjian, and L. Zamick, Phys. Rev. C27, 2782(1983); R. K. Su, S. D. Yang, and T. T. S. Kuo, Phys. Rev. C35, 1539(1987).

[10] M. Baldo, G. Giansiracusa, U. Lombardo, I.Bombaci, and L. S. Ferreira, Nucl. Phys. A583, 589c (1995).

[11] H. R. Jaqaman, A. Z. Mekjian, and L. Zamick,Phys. Rev. C29, 2067 (1984).

[12] B. ter Haar and R. Malfliet, Phys. Rev. Lett. 56, 1237 (1986); Phys. Rep. 149, 207 (1987).

[13] H. Huber, F. Weber and M. K. Weigel, Phys. Rev. C57, 3484 (1998). 
[14] I. Bombaci, T. T. S. Kuo, and U. Lombardo, Phys. Rep. 242, 165 (1994).

[15] P. Grangé, A. Lejeune, M. Martzolff, and J. F. Mathiot, Phys. Rev. C40, 1040 (1989).

[16] A. Lejeune, U. Lombardo, and W. Zuo, Phys. Lett. B477, 45 (2000).

[17] W. Zuo, A. Lejeune, U. Lombardo, and J. F. Mathiot, Nucl. Phys. A706, 418 (2002).

[18] M. Baldo, I. Bombaci, and G. F.Burgio, Astron. Astrophys. 328, 274 (1997).

[19] R. Machleidt, Adv. Nucl. Phys. 19, 189 (1989).

[20] B. S. Pudliner, V. R.Pandharipande, J. Carlson, and R. B. Wiringa, Phys. Rev. Lett. 74, 4396 (1995);B. S. Pudliner, V. R.Pandharipande, J. Carlson, S. C. Pieper, and R. B. Wiringa, Phys. Rev. C56, 1720 (1997).

[21] R. B. Wiringa, V. G. J. Stoks, and R. Schiavilla, Phys. Rev. C51, 38 (1995).

[22] I. Bombaci and U. Lombardo, Phys. Rev. C44, 1892(1991); W. Zuo, I. Bombaci, and U. Lombardo, Phys. Rev. C60, 024605 (1999).

[23] J. P. Jeukenne, A. Lejeune, and C. Mahaux, Phys. Rep. 25C, 83 (1976).

[24] H. Q. Song, M. Baldo, G. Giansiracusa, and U. Lombardo, Phys. Rev. Lett. 81, 1584 (1998).

[25] A. Lejeune, P. Grange, M. Martzolff, and J. Cugnon, Nucl. Phys. A453, 189 (1986).

[26] M. Baldo, I. Bombaci, L. S. Ferreira, G. Giansiracusa, and U. Lombardo, Phys. Lett. B215, 19 (1988).

[27] G. E. Brown, W. Weise, G. Baym and J. Speth, Comm. Nucl. Part. Phys. 17, 39 (1987).

[28] A. M. Lane, Nucl. Phys. 35, 676 (1962).

[29] C. J. Pethick, Rev. Mod. Phys. 64, 1133 (1992).

[30] J. M. Lattimer, C. J. Pethick, M. Prakash, and P. Haensel, Phys. Rev. Lett. 66, 2701 (1991).

[31] H. Huber, F. Weber, and M. K. Weigel, Phys. Lett. B317, 485 (1993); Phys. Rev. C50, 1287 (1994). 
[32] G. H. Bordbar and M. Modarres, Phys. Rev. C57, 714 (1998).

[33] N. Zeldes, Handbook of Nuclear Properties, ed. D. Poenaru and W. Greiner, (Glarendon Press, Oxford, 1996).

[34] D. J. Dean and M. Hjorth-Jensen, Rev. Mod. Phys. 75, 607 (2003) and references therein.

[35] W. Satula and R. Wyss, Phys. Lett. B393, 1 (1997); W. Satula, D. J. Dean, J. Gary, S. Mizutori and W. Nazarewicz, Phys. Lett. B407, 103 (1997).

[36] A. Sedrakian and U. Lombardo, Phys. Rev. Lett. 84, 602 (2000).

[37] U. Lombardo, H.-J. Schulze, and W. Zuo, Phys. Rev. C59, 2927 (1999).

[38] U. Lombardo and W. Zuo, Equation of State of Asymmetric Nuclear Matter, in Isospin Physics in Heavy-Ion Collisions at Intermediate Energies, Eds. B. A. Li and U. W. Schröder, (Nova Science, Huntington, New York, 2001) p.35.

[39] P. Grange, J. Cugnon, and A. Lejeune, Nucl. Phys. A473, 365 (1987).

[40] R. W. Hasse and P. Schuck, Phys. Lett. B179, 313 (1986). 


\section{FIGURES}

FIG. 1. Diagrams of the microscopic TBF adopted for the present calculations, taken from Ref.[15]

FIG. 2. Pressure as function of density for symmetric matter at six values of temperature $T=0,8,10,12,14,16 \mathrm{MeV}$ from the bottom to the top. The solid and dashed curves are the results by using the $A V_{18}$ plus the TBF and the pure $A V_{18}$ two-body force, respectively

FIG. 3. Momentum dependence of the single-particle potential in symmetric nuclear matter at a fixed density $\rho=0.16 \mathrm{fm}^{-3}$ and three different temperatures $T=0,10,20 \mathrm{MeV}$. In the calculations, the TBF is included.

FIG. 4. Proton and neutron single-particle potentials at $k=0$ versus asymmetry parameter $\beta$ for $\rho=0.16 \mathrm{fm}^{-3}$ and $T=10 \mathrm{MeV}$. The solid curves are the results by including the TBF contribution and the dashed ones without the TBF contribution.

FIG. 5. Energy per nucleon $E_{A}(\rho, \beta, T)-E_{A}(\rho, \beta=0, T)$ versus $\beta^{2}$ in the range $0 \leq \beta \leq 1$ for two different densities. The results are obtained by including the TBF.

FIG. 6. Left panel: Symmetry energy as a function of density for three values of temperature. The results are obtained by including the TBF. Right panel: Density dependence of the symmetry energy with the TBF included (solid line) and not included (dashed line) at a fixed temperature $T=10 \mathrm{MeV}$.

FIG. 7. Momentum dependence of the nucleon effective mass in symmetric nuclear matter at three different values of temperature.

FIG. 8. Proton and neutron effective masses as a function of asymmetry parameter obtained by adopting the $A V_{18}$ plus the TBF ( solid curves ) and by using only the $A V_{18}$ two-body force ( dashed curves ). 

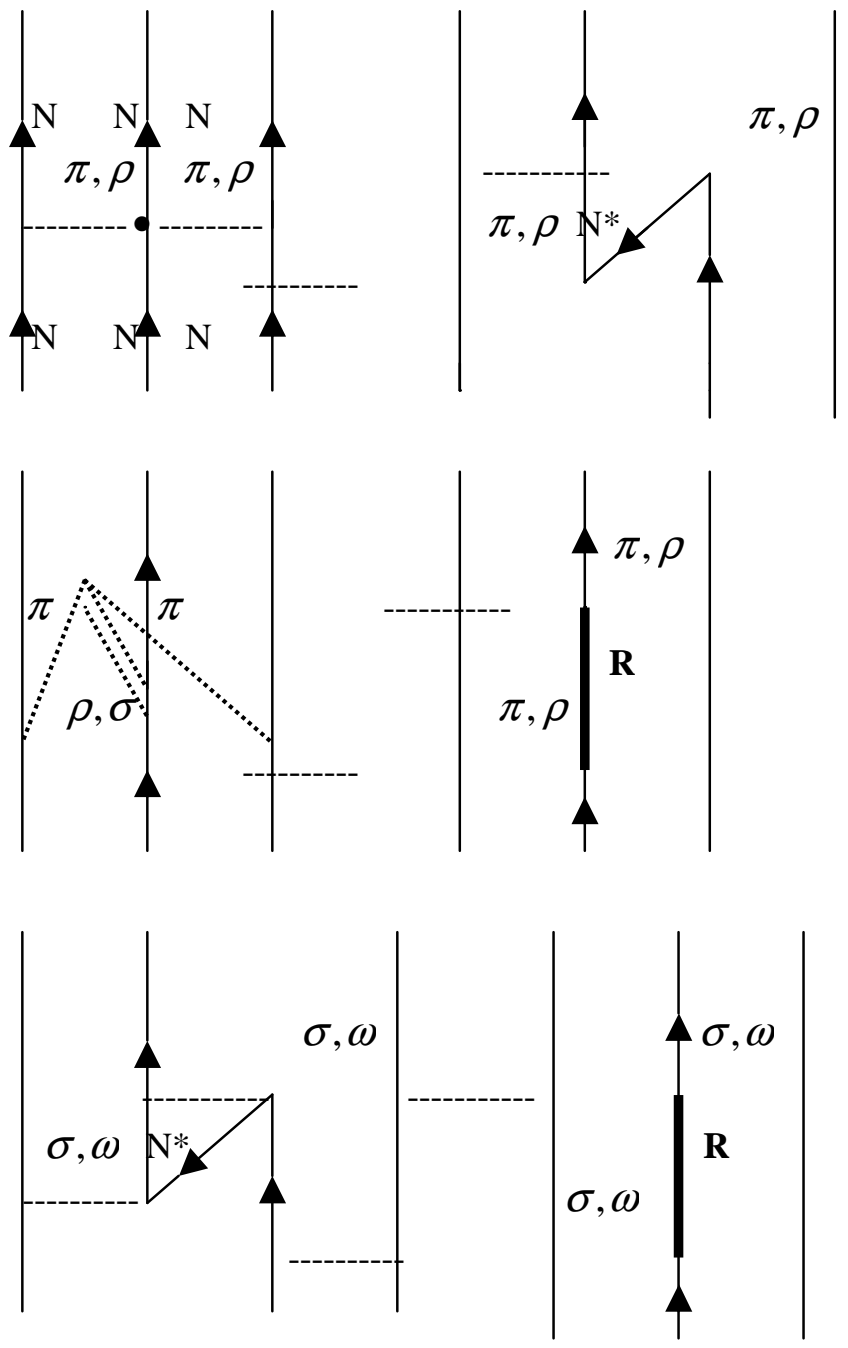

Fig.1 


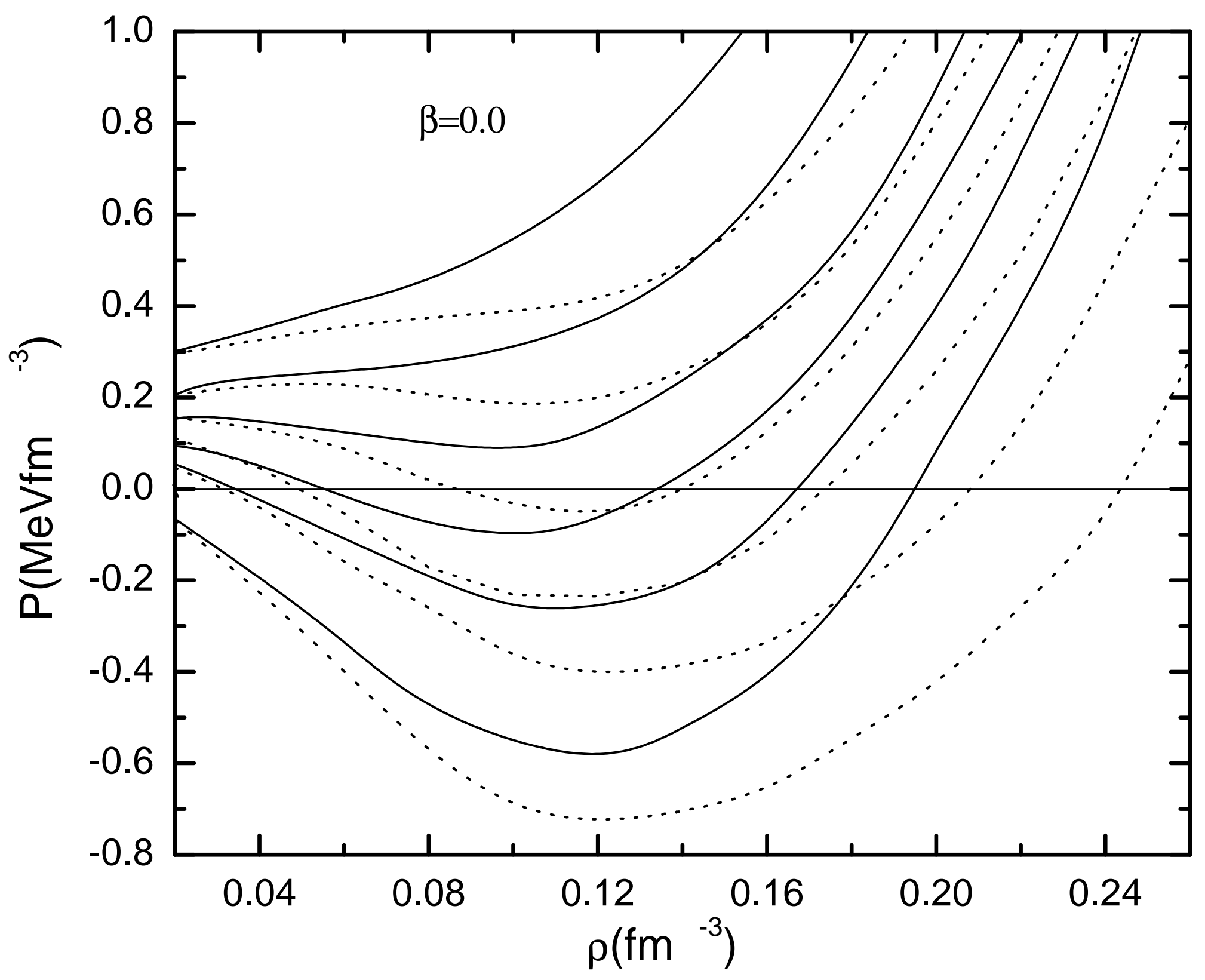

Fig.2 


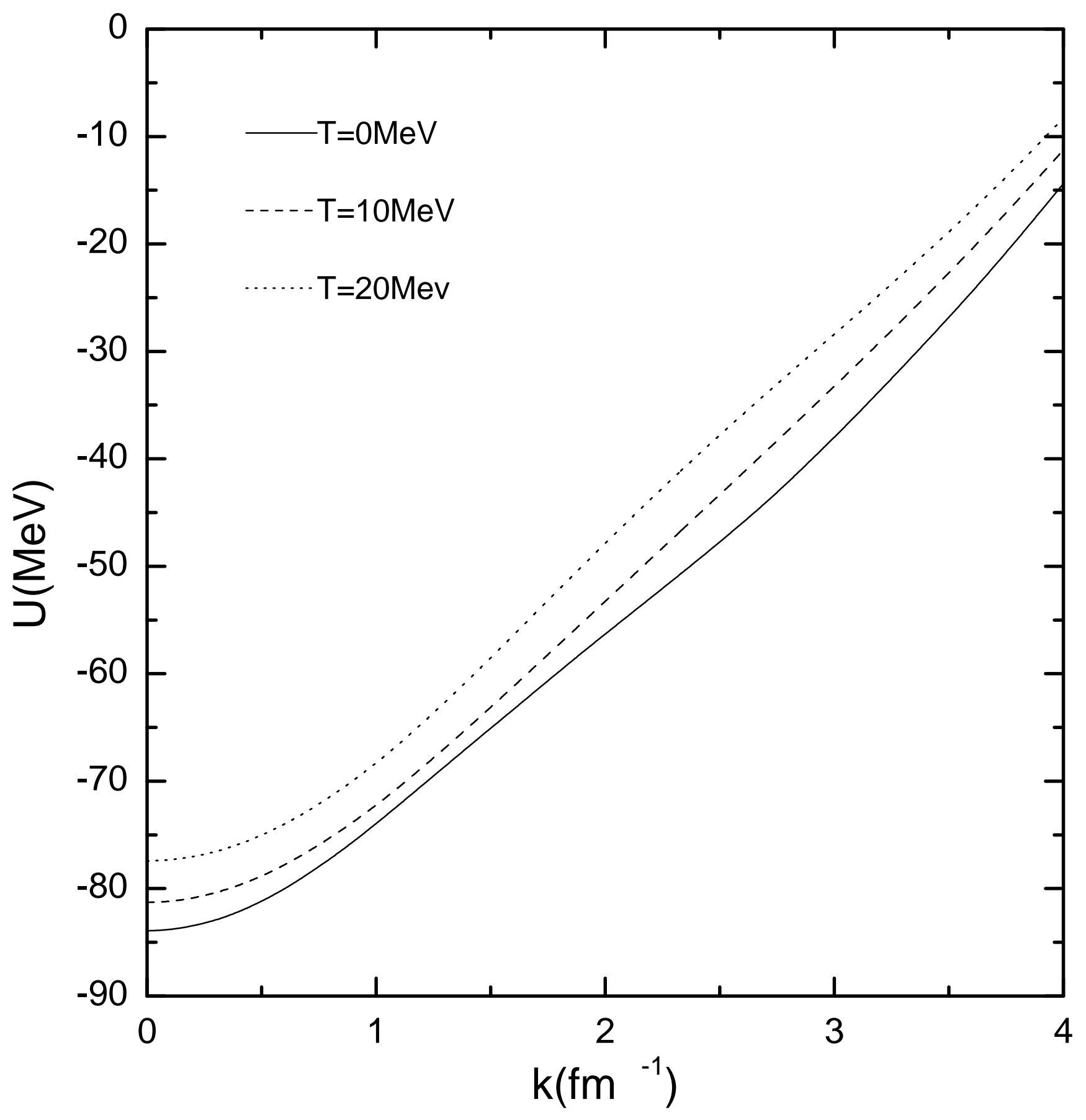

Fig.3 


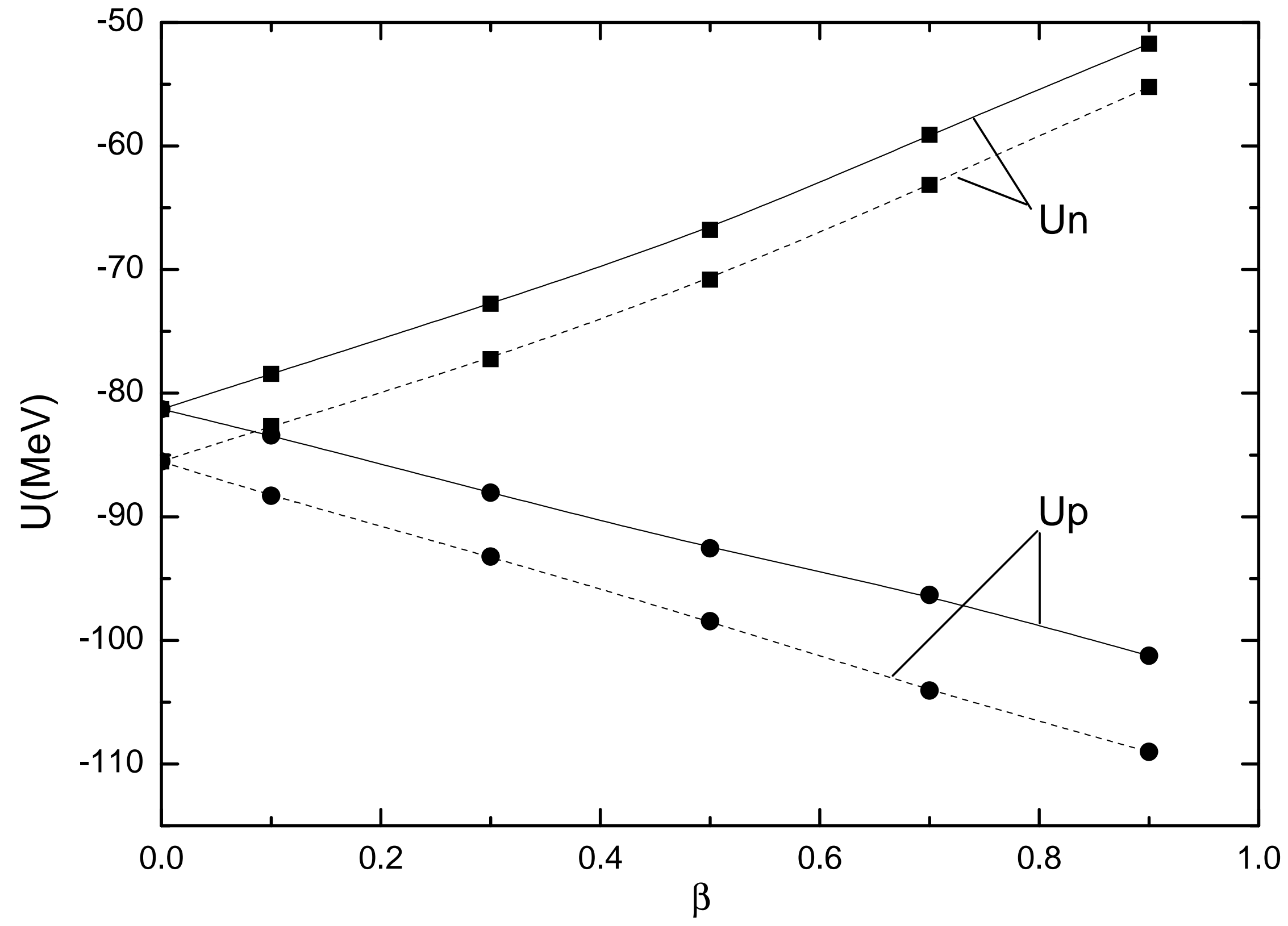

Fig.4 


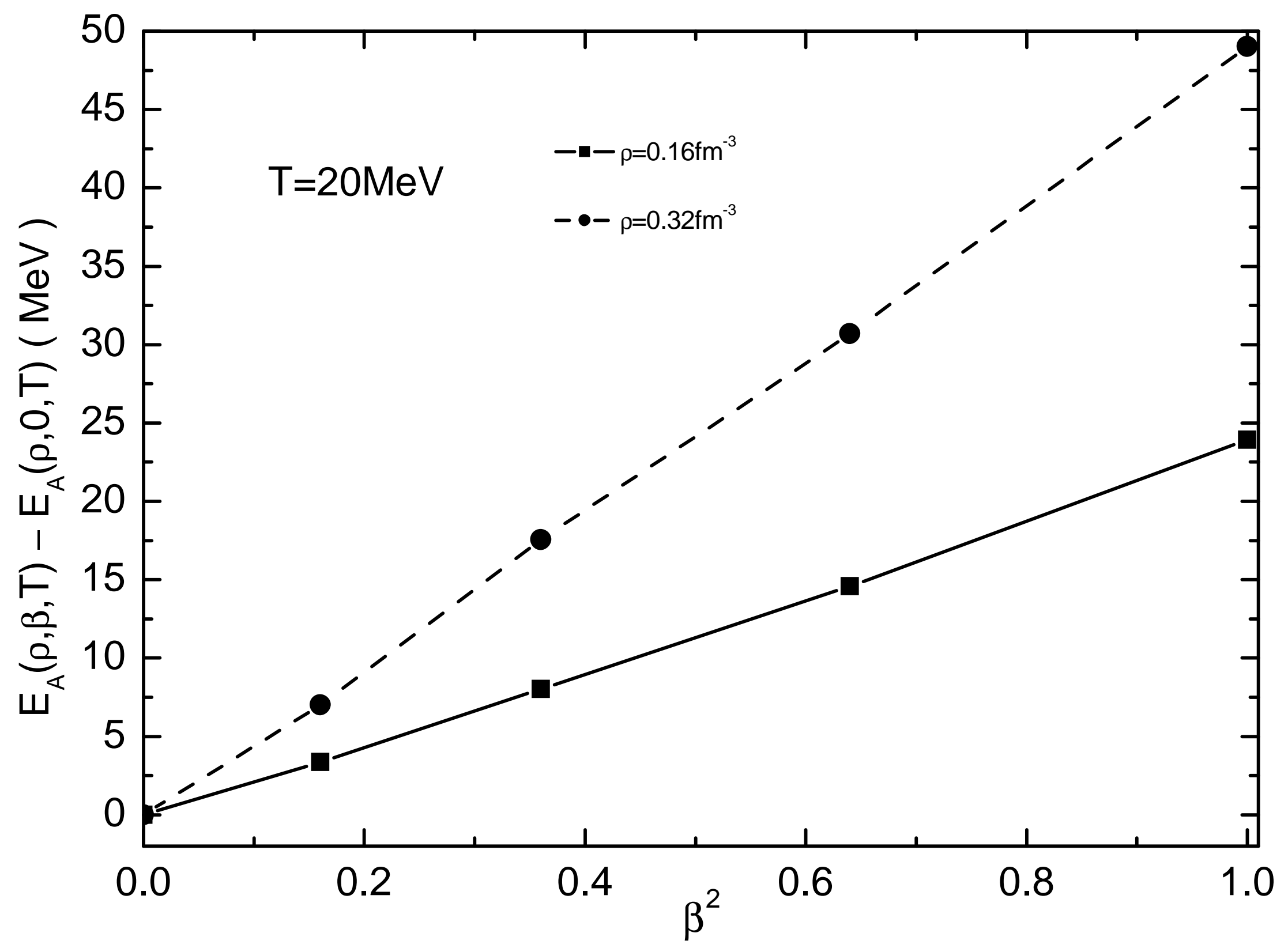

Fig.5 

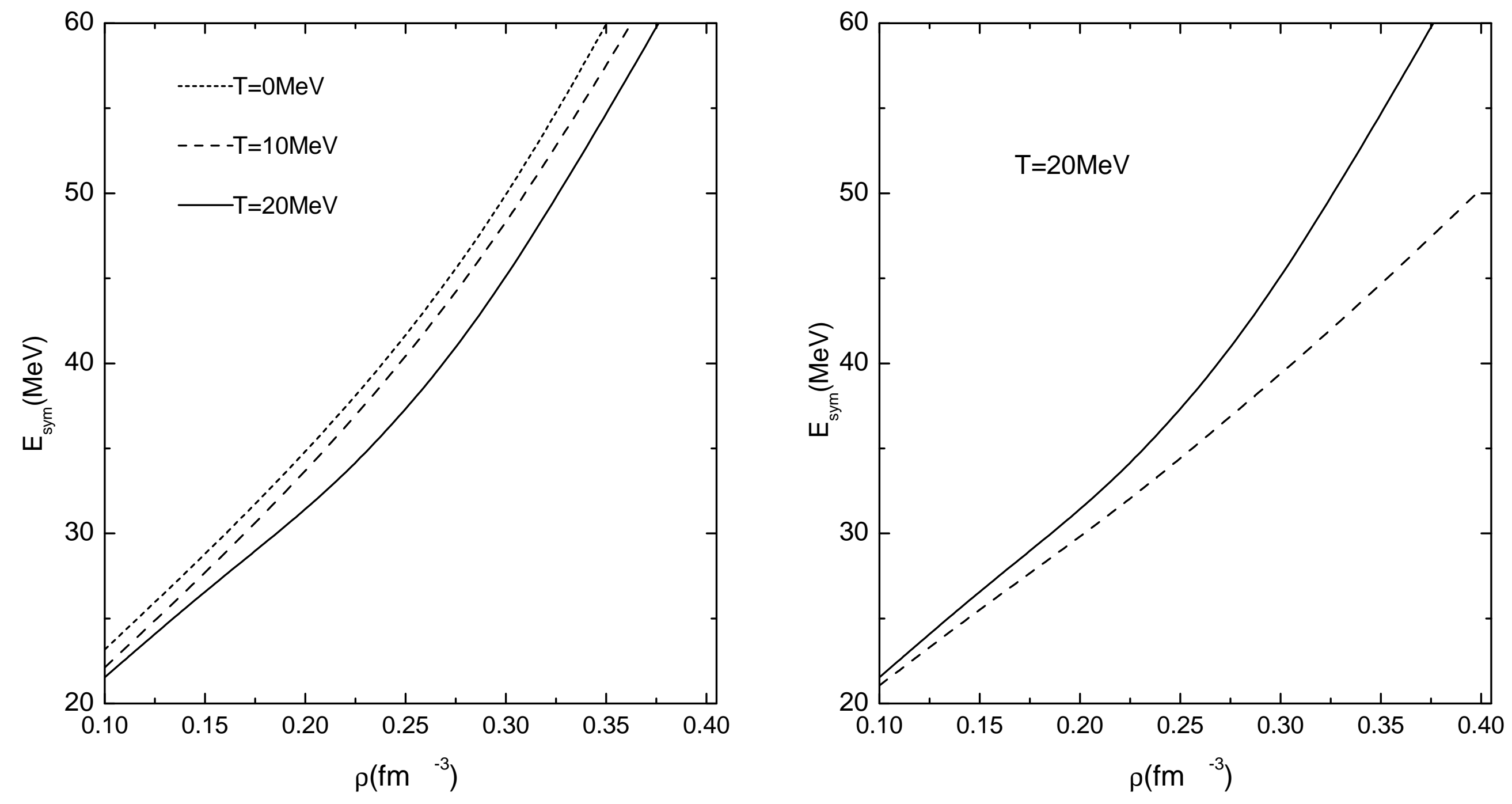

Fig.6 


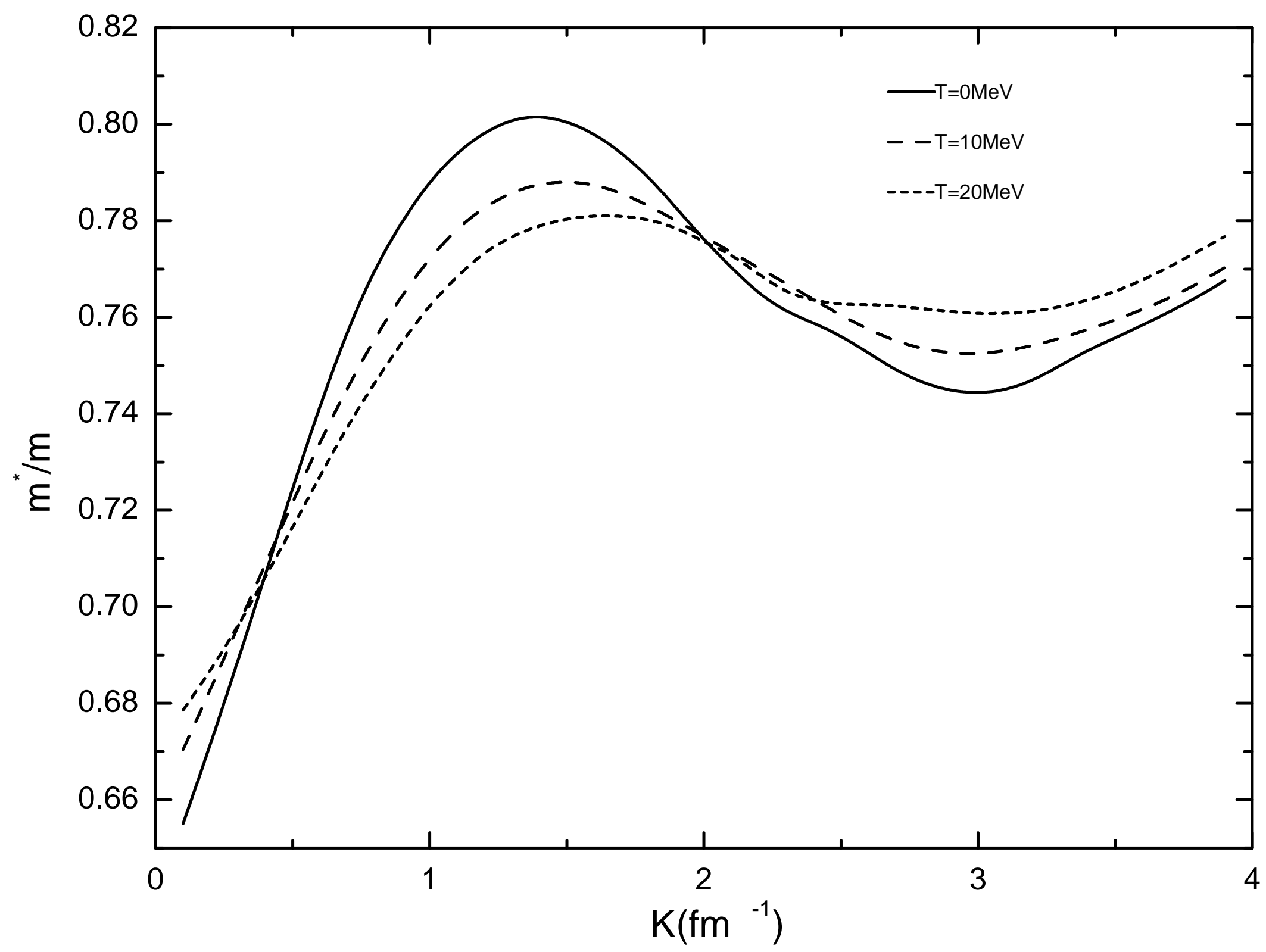

Fig.7 


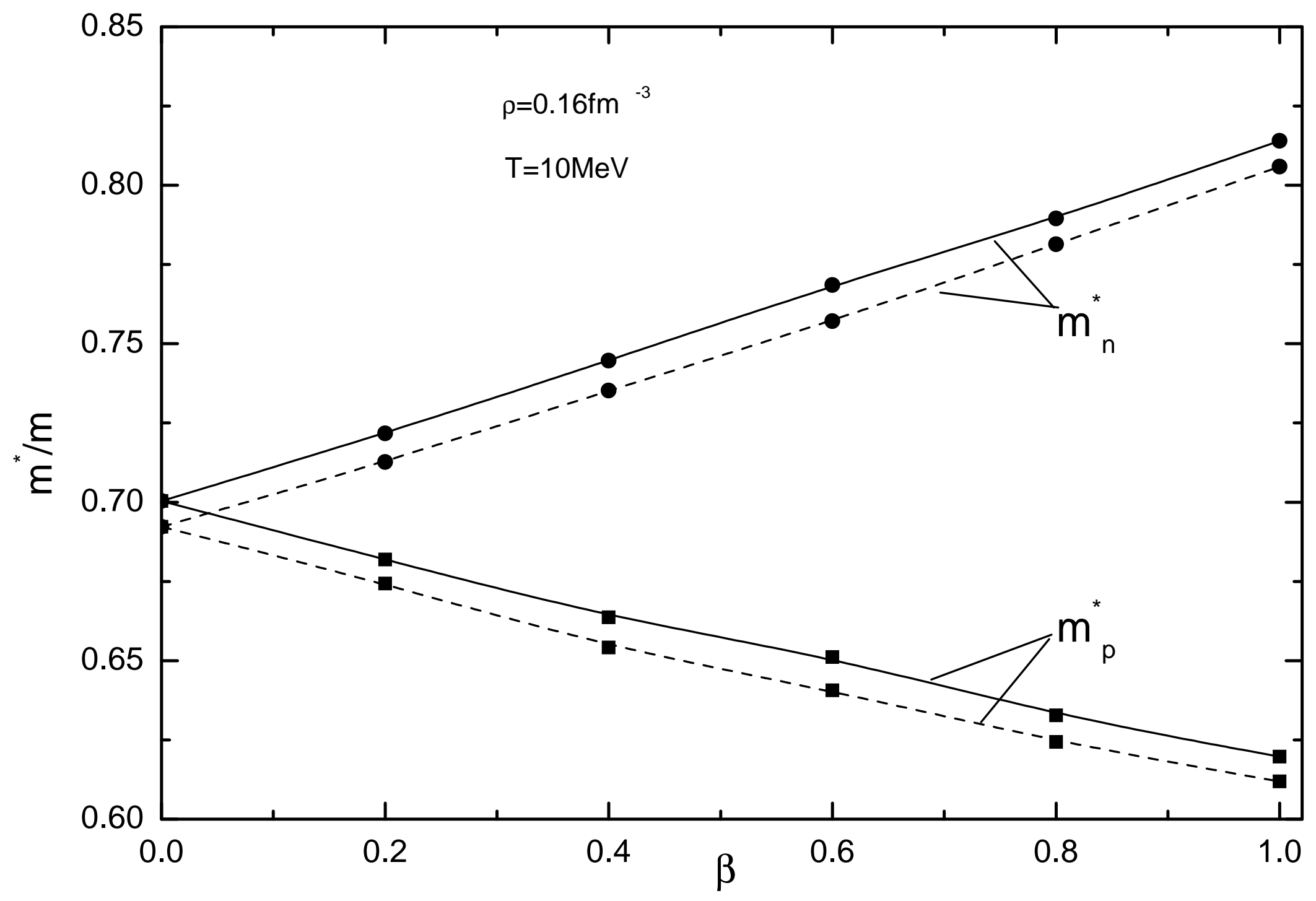

Fig.8 